\title{
The LHC Insertion Magnets
}

\author{
R. Ostojic
}

\begin{abstract}
The Large Hadron Collider comprises eight insertions, four of which are dedicated to the LHC experiments while the others are used for the major collider systems. The various functions of the insertions are fulfilled by a variety of magnet systems, most of them based on the technology of NbTi superconductors cooled by superfluid helium at $1.9 \mathrm{~K}$. A number of stand-alone magnets in the matching sections are operated at $4.5 \mathrm{~K}$, while in the high radiation areas specialized resistive magnets are used. In this paper, we review the concepts underlying the design of the LHC insertions, and report on the design, procurement and testing of the various specialized magnet systems.
\end{abstract}

Index Terms-Insertions, LHC, superconducting magnets.

\section{INTRODUCTION}

$\mathbf{T}$ HE LARGE Hadron Collider, under construction in CERN, is designed to provide proton-proton collisions at center-of-mass energy of $14 \mathrm{TeV}$ and luminosity of $10^{34} \mathrm{~cm}^{-2} \mathrm{~s}^{-1}$ [1]. The LHC consists of two interleaved synchrotron rings, whose main elements are the two-in-one superconducting dipoles and quadrupoles operating in superfluid helium at a temperature of $1.9 \mathrm{~K}$. The collider will be installed in the existing tunnel of $26.7 \mathrm{~km}$ circumference, which until recently housed the LEP collider. The layout of the machine closely mirrors that of LEP, with eight identical arcs, each $2.8 \mathrm{~km}$ long, separated by eight $540 \mathrm{~m}$ long straight sections. Two diametrically opposite insertions in Points 1 and 5 will house the high-luminosity multipurpose experiments ATLAS and CMS, for which considerable civil engineering is required. The more specialized experiments ALICE and LHCb will be installed in the existing caverns of Points 2 and 8 . These insertions will also be equipped with beam injection systems for the two rings. The remaining four insertions will house the other major collider systems: Points 3 and 7 are dedicated to beam collimation, while Points 4 and 6 serve respectively for the RF and beam extraction.

The optics and layout of the LHC insertions have been optimized with respect to their main function and technical and infrastructure constraints specific to each insertion region. Although the general layout of the LHC has not been modified since 1995, the layout of the insertions has been regularly updated. As an example, the CMS high-luminosity insertion has been modified to provide the necessary tuning flexibility for the TOTEM small-angle experiment, and similar features have been implemented in the ATLAS insertion. The collimation systems

Manuscript received September 24, 2001.

The author is with the LHC Division, CERN, 1211 Geneva 23, Switzerland (e-mail: Ranko.Ostojic@cern.ch).

Publisher Item Identifier S 1051-8223(02)03458-9. in Points 3 and 7, designed to limit the beam losses in the superconducting magnets and the background in the experiments, have been considerably reworked to increase their efficiency. Recently, the decision has been taken to displace the superconducting RF cavities in Point 4 from the tunnel to the existing experimental cavern. Consequently, the matching section in Point 4 was rearranged and considerably simplified by reducing the number of quadrupoles and separation dipoles and standardizing their types. All these modifications were necessary to resolve the functional or installation bottlenecks that were identified with the advance of the equipment design and its integration in the tunnel.

In spite of functional differences, all insertions contain, moving from the arc toward the middle of an insertion, a dispersion suppressor followed by a matching section, a section of separation dipoles, and in the experimental insertions, the final focus system (low- $\beta$ triplet). Most of the magnets in the insertions are based on the state-of-the-art superconducting magnet technology of NbTi superconductors cooled by superfluid helium at $1.9 \mathrm{~K}$. However, a number of stand-alone quadrupoles in the matching sections, as well as the separation dipoles, are operated at $4.5 \mathrm{~K}$. In the high radiation areas specialized resistive magnets are used. The areas in between the magnets house the beam injection and extraction systems, collimators and the RF system and beam instrumentation, and in the experimental insertions the large detector magnets. All these systems have to be integrated with the general collider services in the tight space of the LHC tunnel.

A significant part of the magnet systems in the insertions is supplied by accelerator laboratories world-wide as part of the contributions of respective nonmember countries to the LHC. In view of the complicated interfaces, advanced stage of equipment design and tight budgets, close collaboration and frequent contacts between laboratories have been established, which have allowed, together with modern engineering document management tools, efficient monitoring of progress and close configuration control.

Following extensive R\&D and technical validation of the major collider systems in CERN and collaborating laboratories [2], industrial contracts for the procurement of the superconducting and resistive magnets for the LHC insertions have been awarded, or their construction started in laboratory production facilities. Although first deliveries are expected already by the end of 2001, most of the magnets will be delivered following their installation sequence, which is foreseen to start in Autumn 2003. The commissioning of the first insertion in Point 8 is planned for Spring 2004 as part of the Octant 7-8 test. The remaining systems will be installed and the ring closed by the end of 2005. 




Fig. 1. Schematic layout of the LHC low- $\beta$ triplet.

TABLE I

MAIN PARAMETERS OF THE MQXA LOW- $\beta$ QUADRUPOLE

\begin{tabular}{lcc}
\hline \hline Coil inner diameter & \multicolumn{2}{c}{$70 \mathrm{~mm}$} \\
Magnetic length & $6.37 \mathrm{~m}$ \\
Operating temperature & \multicolumn{2}{c}{$1.9 \mathrm{~K}$} \\
Nominal gradient & $215 \mathrm{~T} / \mathrm{m}$ \\
Nominal current & $7149 \mathrm{~A}$ \\
Peak field in coil & \multicolumn{2}{c}{$8.6 \mathrm{~T}$} \\
Quench field & \multicolumn{2}{c}{$10.7 \mathrm{~T}$} \\
Stored energy & \multicolumn{2}{c}{$2300 \mathrm{~kJ}$} \\
Inductance & \multicolumn{2}{c}{$90.1 \mathrm{mH}$} \\
\hline & Cable 1 & Cable 2 \\
Width (mm) & 11 & 11 \\
Mid-thickness (mm) & 1.487 & 1.340 \\
Keystone angle (deg.) & 2.309 & 1.319 \\
No of strands & 27 & 30 \\
Strand dia. (mm) & 0.815 & 0.735 \\
Cu/SC Ratio & 1.2 & 1.9 \\
Filament dia. ( $\mu \mathrm{m}$ ) & 10 & 10 \\
$j_{c}\left(\mathrm{~A} / \mathrm{mm}^{2}, 4.2 \mathrm{~K}\right.$ and $\left.6 \mathrm{~T}\right)$ & 2200 & 2160 \\
\hline
\end{tabular}

\section{The Final Focus Magnets}

The low- $\beta$ triplet, Fig. 1, is located at $23 \mathrm{~m}$ on each side of the interaction point, and is composed of four single aperture quadrupoles with a coil aperture of $70 \mathrm{~mm}$. It is cooled with superfluid helium at $1.9 \mathrm{~K}$ using an external heat exchanger system capable of extracting up to $10 \mathrm{~W} / \mathrm{m}$ of power deposited in the coils by the secondaries emanating from the $p$-p collisions. Two types of quadrupoles are used in the triplet, $6.6 \mathrm{~m}$ long MQXA magnets designed and developed by KEK (Japan), and $5.7 \mathrm{~m}$ long MQXB magnets designed and built by FNAL (USA). The magnets are powered in series with $7 \mathrm{kA}$, with an additional inner loop of $5 \mathrm{kA}$ for the MQXB magnets. Together with the orbit correctors and multipole spool pieces supplied by CERN, the low- $\beta$ quadrupoles are completed in their cold masses and cryostated by FNAL. The cryogenic feedboxes, providing a link to the cryogenic distribution line and power converters, are designed and built by LBNL (USA).

Alongside the LHC main dipoles, the high-gradient, wide-aperture low- $\beta$ quadrupoles are the most demanding magnets in the collider. They must operate reliably at $215 \mathrm{~T} / \mathrm{m}$, sustain extremely high heat loads in the coils and radiation dose during their lifetime, and have a very good field quality within the $63 \mathrm{~mm}$ aperture of the cold bore. The design of the MQXA quadrupole is based on a four-layer coil using $11 \mathrm{~mm}$ wide Rutherford-type graded NbTi cables. The coils are wound and cured in two double layers, and are assembled using $10 \mathrm{~mm}$ wide spacer-type collars. The pre-stress in the coils and their rigidity is provided by the yoke structure, which consists of
TABLE II

MAIN PARAMETERS OF THE MQXB LOW- $\beta$ QUADRUPOLE

\begin{tabular}{lcc}
\hline \hline Coil inner diameter & \multicolumn{2}{c}{$70 \mathrm{~mm}$} \\
Magnetic length & $5.5 \mathrm{~m}$ \\
Operating temperature & \multicolumn{2}{c}{$1.9 \mathrm{~K}$} \\
Nominal gradient & \multicolumn{2}{c}{$215 \mathrm{~T} / \mathrm{m}$} \\
Nominal current & \multicolumn{2}{c}{$11950 \mathrm{~A}$} \\
Peak field in coil & \multicolumn{2}{c}{$7.7 \mathrm{~T}$} \\
Quench field & \multicolumn{2}{c}{$9.2 \mathrm{~T}$} \\
Stored energy & \multicolumn{2}{c}{$1360 \mathrm{~kJ}$} \\
Inductance & \multicolumn{2}{c}{$19.1 \mathrm{mH}$} \\
\hline & Cable 1 & Cable 2 \\
Width (mm) & 15.4 & 15.4 \\
Mid-thickness (mm) & 1.456 & 1.146 \\
Keystone angle (deg.) & 1.079 & 0.707 \\
No of strands & 37 & 46 \\
Strand dia. (mm) & 0.808 & 0.650 \\
Cu/SC Ratio & 1.3 & 1.8 \\
Filament dia. ( $\mu \mathrm{m}$ ) & 6 & 6 \\
$j_{\text {c }}\left(\mathrm{A} / \mathrm{mm}^{2}, 4.2 \mathrm{~K}\right.$ and $\left.5 \mathrm{~T}\right)$ & 2750 & 2750 \\
\hline \hline
\end{tabular}

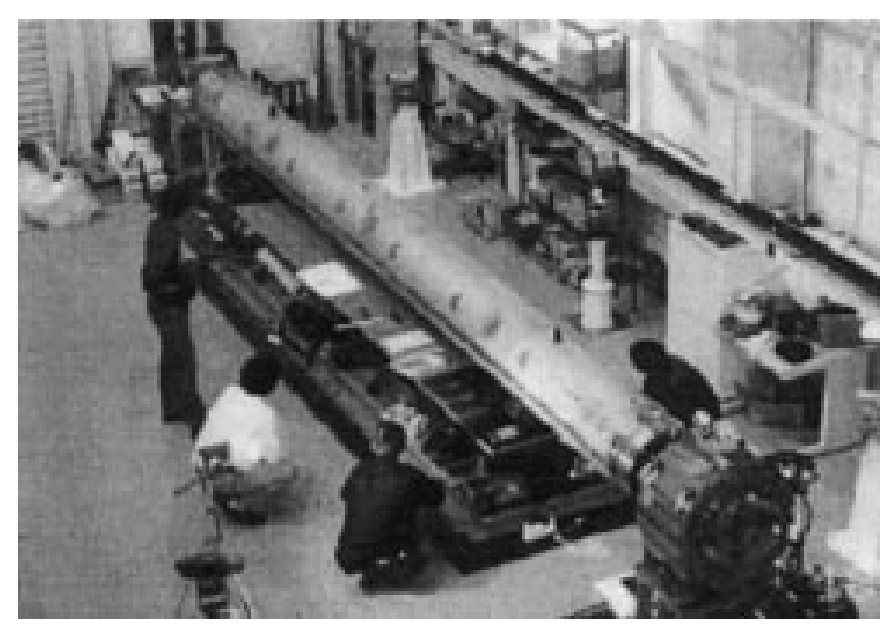

Fig. 2. Prototype MQXA low- $\beta$ quadrupole on the warm test bench in KEK.

horizontally split laminations keyed at the mid-plane. The main parameters of the magnet are given in Table I.

The MQXB design features a two-layer coil, with each layer individually wound using a $15.4 \mathrm{~mm}$ wide Rutherford-type NbTi cable. The coils are assembled using free-standing collars, which provide the pre-stress and reacts the magnetic forces. The collared assembly is aligned in the yoke structure with precision keys, and the magnet enclosed in a stainless steel helium vessel consisting of half-shells welded at the pole plane. The design parameters of the magnet are given in Table II.

In order to validate their design choices, KEK and Fermilab have launched in 1996 comprehensive R\&D programs comprising a number of short model magnets, refs. [3] and [4]. Both programs were successful in fulfilling the design goals and demonstrating the LHC operational requirements, following which full-length prototype magnets were constructed.

The $6.6 \mathrm{~m}$ long prototype MQXA quadrupole is shown in Fig. 2 during reception in KEK. The magnet was tested in the newly built $1.9 \mathrm{~K}$ vertical test facility in KEK in April 2001, and surpassed the ultimate gradient in LHC of $215 \mathrm{~T} / \mathrm{m}$ in two training quenches. The training was confirmed after a thermal cycle, when the magnet reached $230 \mathrm{~T} / \mathrm{m}$ in two quenches. In 


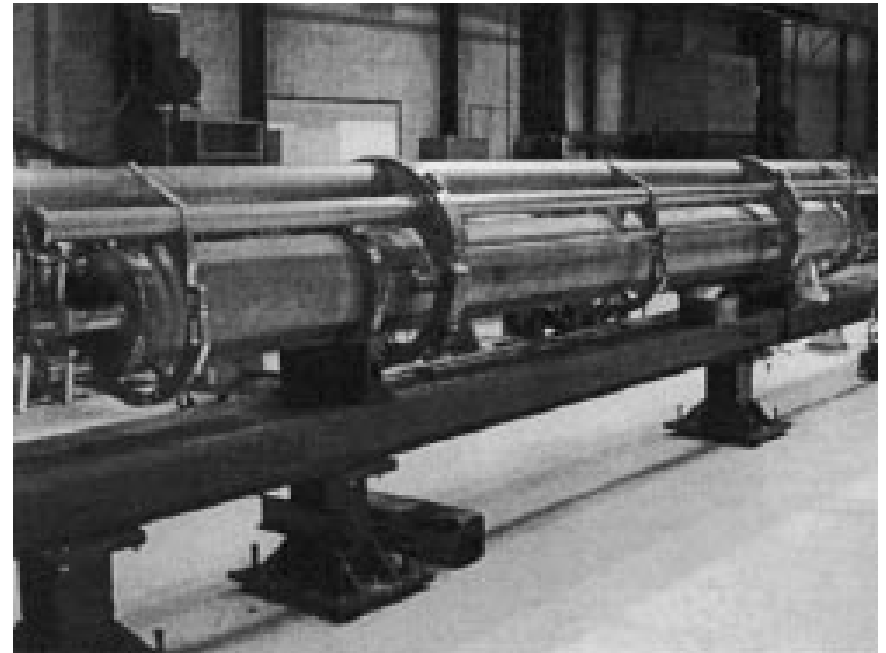

Fig. 3. Prototype MQXB low- $\beta$ quadrupole equipped with the external $1.9 \mathrm{~K}$ heat exchanger ready for cryostating in Fermilab.

Fig. 3, the $5.7 \mathrm{~m}$ long MQXB prototype is shown ready for cryostating. This magnet was tested in the upgraded $1.9 \mathrm{~K}$ horizontal test station in FNAL in May 2001, and reached the target for quench training of $230 \mathrm{~T} / \mathrm{m}$ in eight quenches, confirmed after a thermal cycle. On the basis of these excellent results, the series fabrication of the low- $\beta$ quadrupoles has been launched. In total, 16 MQXA quadrupoles will be supplied by KEK, built in Japanese industry, and 16 MQXB quadrupoles will be built in Fermilab. The first triplet is expected in CERN by the end of 2002.

An important result of both $R \& D$ programs, confirmed by the quadrupole prototypes, is that the field quality is considerably better than initially expected. As a result, the number and strength of the multipole correctors could be reduced and their arrangement simplified. In view of the fact that the low- $\beta$ quadrupoles govern the dynamic aperture and luminosity of the LHC at top energy, these results give confidence in achieving the design performance of the collider.

\section{SEPARATION DipOlES}

The separation dipoles are used in several insertions to change the beam separation from the nominal $194 \mathrm{~mm}$ in the LHC arcs. In the experimental insertions the pair of D1-D2 dipoles brings the two beams onto a colliding orbit at the interaction point. To reduce the beam-beam effects, the first separation dipole D1 is placed immediately upstream of the low- $\beta$ triplet. In the high-luminosity insertions, high radiation levels are expected and more robust resistive magnets (MBXW) are used. In the ALICE and LHCb insertions, D1 is a stronger superconducting magnet (MBX) allowing more space for the injection systems. In all cases, the $\mathrm{D} 2$ separation dipole (MBR) is a twin-aperture superconducting magnet. In the cleaning insertions, the pair of D3-D4 dipoles separates the beams to $222 \mathrm{~mm}$ to accommodate the collimators, while in the RF insertion the beam separation is $420 \mathrm{~mm}$ required for the superconducting cavities. The radiation levels in Points 3 and 7 require the use of resistive dipoles
TABLE III

MAIN PARAMETERS OF THE MBX, MBR, AND MBRS SUPERCONDUCTING SEPARATION DIPOLES

\begin{tabular}{lc}
\hline \hline Coil inner diameter & $80 \mathrm{~mm}$ \\
Magnetic length & $9.45 \mathrm{~m}$ \\
Operating temperature & $1.9 \mathrm{~K}(\mathrm{MBX})$ \\
& $4.5 \mathrm{~K}$ (MBR, MBRS) \\
Aperture separation & $-(\mathrm{MBX})$ \\
& $188 / 194 \mathrm{~mm}(\mathrm{MBR})$ \\
Nominal field & $420 \mathrm{~mm}(\mathrm{MBRS})$ \\
Nominal current & $3.8 \mathrm{~T}$ \\
& $5750 \mathrm{~A} \mathrm{(MBX,} \mathrm{MBRS)}$ \\
Peak field in coil & $6050 \mathrm{~A}(\mathrm{MBR})$ \\
Quench field & $4.2 \mathrm{~T}$ \\
Stored energy per aperture & $4.8 \mathrm{~T}$ \\
Inductance per aperture & $470 \mathrm{~kJ}$ \\
\hline Cable width $(\mathrm{mm})$ & $25.8 \mathrm{mH}$ \\
Mid-thickness $(\mathrm{mm})$ & 9.73 \\
Keystone angle $(\mathrm{deg})$ & 1.166 \\
No of strands & 1.2 \\
Strand dia. $(\mathrm{mm})$ & 30 \\
Cu/SC Ratio & 0.648 \\
Filament dia. $(\mu \mathrm{m})$ & 1.8 \\
$j_{c}\left(\mathrm{~A} / \mathrm{mm}^{2}, 4.2 \mathrm{~K}\right.$ and $\left.5 \mathrm{~T}\right)$ & 6 \\
\hline \hline
\end{tabular}



Fig. 4. The first series D1 separation dipole ready for tests in BNL.

(MBW), while superconducting dipoles MBR and MBRS are used in Point 4 as D4 and D3, respectively.

The MBX, MBR, and MBRS dipoles are designed and built by BNL (USA) on the basis of the RHIC lattice dipole, and feature a $10 \mathrm{~m}$ long, $80 \mathrm{~mm}$ aperture coil. While MBX and MBRS are single aperture cold masses, essentially identical to the RHIC lattice dipole, MBR is a two-in-one magnet which considerably deviates from the RHIC design. All magnets operate at a field of up to $3.8 \mathrm{~T}$, at a temperature of $1.9 \mathrm{~K}$ for MBX and of $4.5 \mathrm{~K}$ for MBR and MBRS. The main parameters of the superconducting separation dipoles are given in Table III.

To validate the design of the twin-aperture MBR magnet, BNL has built and tested two $3 \mathrm{~m}$ long prototypes, which showed very satisfactory quench performance and field quality [5]. The series production of these magnets has started in BNL. All five MBX cold masses have been completed and the first unit assembled in its cryostat, Fig. 4. The first MBR cold mass, Fig. 5, has also been built and is ready for cryostating. The first 


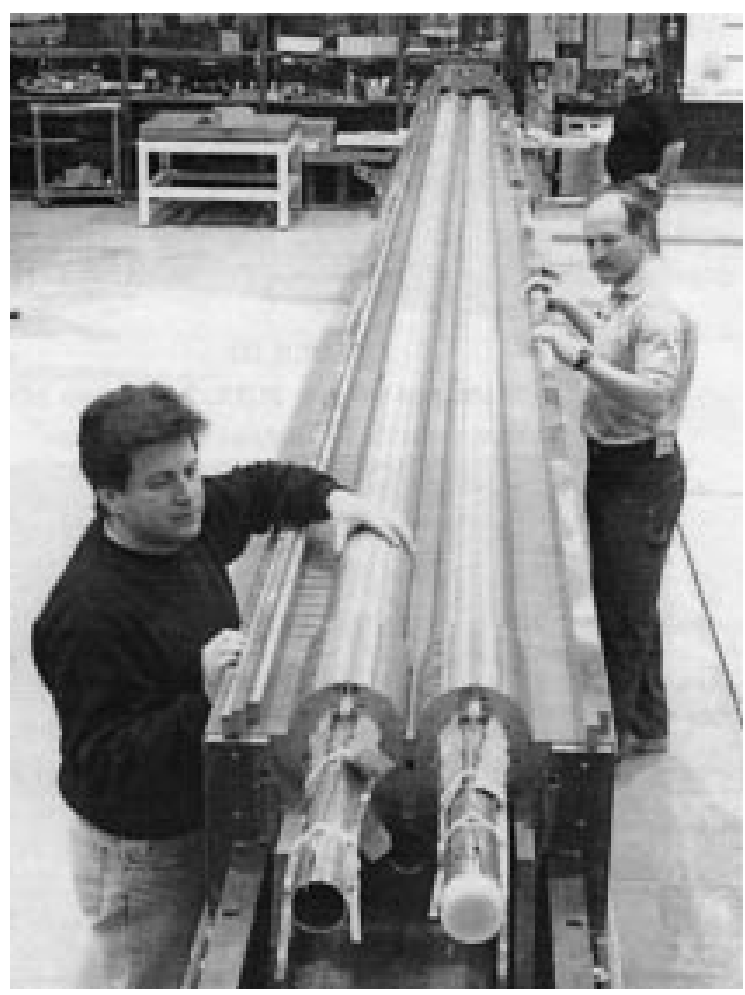

Fig. 5. Assembly of the first series MBR separation dipole in BNL.

separation dipoles are expected to be delivered to CERN by the end of 2001.

\section{DisPersion SuPPRESSORS AND MATCHING SECTIONS}

The optics flexibility of the LHC insertions is provided by the individually powered quadrupoles in the dispersion suppressors and the matching sections. All dispersion suppressors comprise four half cells, with two dipoles per half cell, identical to those in the arcs. The suppressors are an integral part of the arc cryostat, and share the powering and cryogenic infrastructure. The matching sections in general consist of stand alone quadrupoles, but the number and parameters of the magnets are specific for each insertion. They also comprise four half cells, except in Points 4 and 6, where the matching section has respectively 3 and 2 quadrupoles. Furthermore, in the cleaning insertions, specialized resistive quadrupoles are used in the part of the matching section located in high radiation areas.

CERN has developed two superconducting quadrupoles for the dispersion suppressors and matching sections, one featuring a $56 \mathrm{~mm}$ aperture coil (MQM), and the other an enlarged, $70 \mathrm{~mm}$ aperture coil (MQY). Both quadrupoles use narrow cables, so that the nominal current is less than $6 \mathrm{kA}$, substantially simplifying the warm and cold powering circuits. These magnets are assembled in several different types of cold masses, comprising one or two quadrupoles and one or three orbit correctors, and range in length from $5.2 \mathrm{~m}$ to $11.6 \mathrm{~m}$. Similarly to the LHC main dipoles, the cold masses are assembled using two welded half-shells, which provide the rigidity and alignment of the magnets and serve as a helium vessel.

The MQM quadrupole, Fig. 6, consists of two identical, independently powered apertures, which are assembled together in a

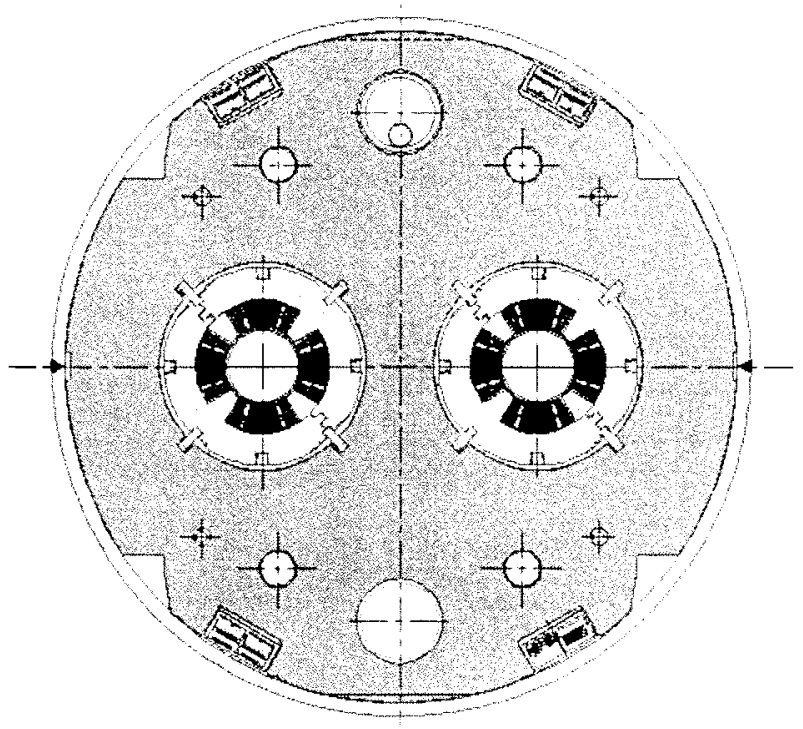

Fig. 6. Cross-section of the MQM matching quadrupole.

TABLE IV

MAIN PARAmeters OF THE MQM TWIN-APERTURE MATCHING QUADRUPOLES

\begin{tabular}{lc}
\hline Coil inner diameter & $56 \mathrm{~mm}$ \\
Magnetic length & $2.4 / 3.4 / 4.8 \mathrm{~m}$ \\
Operating temperature & $1.9 \mathrm{~K}$ \\
Nominal gradient & $200 \mathrm{~T} / \mathrm{m}$ \\
Nominal current & $5390 \mathrm{~A}$ \\
Peak field in coil & $6.3 \mathrm{~T}$ \\
Quench field & $7.8 \mathrm{~T}$ \\
Stored energy per aperture & $64.3 \mathrm{~kJ} / \mathrm{m}$ \\
Inductance per aperture & $4.44 \mathrm{mH} / \mathrm{m}$ \\
\hline Cable width (mm) & 8.80 \\
Mid-thickness (mm) & 0.84 \\
Keystone angle (deg.) & 0.91 \\
No of strands & 36 \\
Strand dia. (mm) & 0.475 \\
Cu/SC Ratio & 1.75 \\
Filament dia. ( $\mu m$ ) & 6 \\
$j_{c}\left(\right.$ A $/$ mm ${ }^{2}, 4.2 \mathrm{~K}$ and $\left.5 \mathrm{~T}\right)$ & 2800 \\
\hline \hline
\end{tabular}

two-in-one yoke structure. The coils are made using an $8.8 \mathrm{~mm}$ wide Rutherford-type NbTi cable wound as a double layer and cured in a single cycle. The design was optimized for the highest transfer function and operational margin, and a geometrical $b_{6}$ multipole which partially compensates the $b_{6}$ term due to persistent currents. The coils are assembled using $21 \mathrm{~mm}$ wide collars, locked with four full-length tapered keys, which provide the necessary compressive stress in the coils and withstand the magnetic forces. The collared apertures are assembled together using single-piece iron laminations, very similar in design to the LHC arc quadrupole, so that the cooling and busbar systems in the dispersion suppressors are fully compatible. Three versions of the MQM quadrupole are required for the LHC, with magnetic lengths of $2.4 \mathrm{~m}, 3.4 \mathrm{~m}$ and $4.8 \mathrm{~m}$. The main parameters of the quadrupole are listed in Table IV.

The MQM design was validated on two single aperture and a twin-aperture $1 \mathrm{~m}$ long magnets [6]. All magnets showed very good training behavior, with first training quenches at $210 \mathrm{~T} / \mathrm{m}$, above the nominal gradient in the LHC. The magnets trained to 


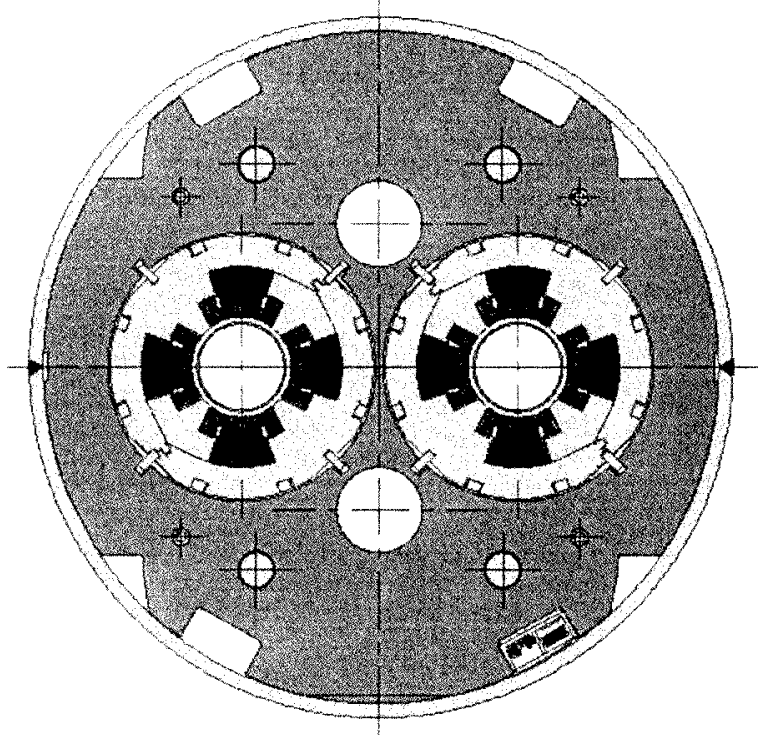

Fig. 7. Cross-section of the MQY wide-aperture matching quadrupole.

the conductor limit of $255 \mathrm{~T} / \mathrm{m}$ in less than ten quenches and retained their training state after a thermal cycle. A recently tested short prototype of the magnet, featuring a superconducting cable with final dimensions, had its first training quench at $240 \mathrm{~T} / \mathrm{m}$, additionally confirming the soundness of the design. Protection studies on the model magnets showed that the designed heaters covering the outer layer of the coil provide redundant protection. The field quality of all magnets was within specifications. The contract for the supply of 94 MQM quadrupoles has been adjudicated to Tesla Engineering (UK), and the first pre-series magnets are expected in the beginning of 2002.

Similarly to MQM, the MQY wide-aperture quadrupole consists of two $3.6 \mathrm{~m}$ long individually powered apertures assembled in a common yoke structure, Fig. 7. The $70 \mathrm{~mm}$ aperture coils have four layers. The inner two layers are wound and cured in a single cycle using two $8.3 \mathrm{~mm}$ wide Rutherford-type graded NbTi cables, with the transition between the cables in the middle of the second layer. The outer two layers are wound and cured using only the smaller cable. The coils are assembled using a self-supporting collar system, consisting of two $22 \mathrm{~mm}$ wide collars which are locked with eight full-length tapered keys. As in the LHC arc quadrupoles and MQM quadrupoles, the collared coils are assembled in the two-in-one yoke structure using four full-length keys at the poles, which center the assemblies once the laminations are in place and locked with dowel pins. The main parameters of the quadrupole are given in Table V. Following the successful model program [7], the contract for the supply of $22 \mathrm{MQY}$ quadrupoles has been adjudicated to ACCEL Instruments (Germany), and first pre-series magnets are expected in the beginning of 2002.

\section{Resistive MAGNETS}

A number of specialized resistive magnets will be installed in those areas of the insertions where the radiation levels are expected to be high. In the beam cleaning insertions, apart from the MBW dipoles which provide beam separation, twin-aperture
TABLE V

Main Parameters of THE MQY Two-IN-ONE Wide APERTURE MatChING QUADRUPOLE

\begin{tabular}{lcc}
\hline \hline Coil inner diameter & $70 \mathrm{~mm}$ \\
Magnetic length & $3.4 \mathrm{~m}$ \\
Operating temperature & \multicolumn{2}{c}{$4.5 \mathrm{~K}$} \\
Nominal gradient & $160 \mathrm{~T} / \mathrm{m}$ \\
Nominal current & \multicolumn{2}{c}{$3610 \mathrm{~A}$} \\
Peak field in coil & \multicolumn{2}{c}{$6.1 \mathrm{~T}$} \\
Quench field & \multicolumn{2}{c}{$7.5 \mathrm{~T}$} \\
Stored energy per aperture & $141 \mathrm{~kJ} / \mathrm{m}$ \\
Inductance per aperture & $21.7 \mathrm{mH} / \mathrm{m}$ \\
\hline & Cable 1 & Cable 2 \\
Width (mm) & 8.3 & 8.3 \\
Mid-thickness (mm) & 1.285 & 0.845 \\
Keystone angle (deg.) & 2.16 & 1.05 \\
No of strands & 22 & 34 \\
Strand dia. (mm) & 0.735 & 0.475 \\
Cu/SC Ratio & 1.25 & 1.75 \\
Filament dia. ( $\mu \mathrm{m}$ ) & 6 & 6 \\
$j_{c}\left(\mathrm{~A} / \mathrm{mm}^{2}, 4.2 \mathrm{~K}\right.$ and $\left.5 \mathrm{~T}\right)$ & 2670 & 2800 \\
\hline \hline
\end{tabular}

TABLE VI

MAIN PARAMETERS OF THE RESISTIVE MAGNETS IN THE LHC INSERTIONS

\begin{tabular}{lccc}
\hline \hline & MQW & MBW & MBWX \\
\hline Aperture/gap & $46 \mathrm{~mm}$ & $52 \mathrm{~mm}$ & $63 \mathrm{~mm}$ \\
Nominal strength & $35 \mathrm{~T} / \mathrm{m}$ & $1.42 \mathrm{~T}$ & $1.38 \mathrm{~T}$ \\
Magnetic length & $3.108 \mathrm{~m}$ & $3.4 \mathrm{~m}$ & $3.4 \mathrm{~m}$ \\
Nominal current & $710 \mathrm{~A}$ & $726 \mathrm{~A}$ & $723 \mathrm{~A}$ \\
Power & $20 \mathrm{~kW}$ & $35 \mathrm{~kW}$ & $35 \mathrm{~kW}$ \\
Overall weight & 11 tons & 20 tons & 20 tons \\
\hline \hline
\end{tabular}

MQW quadrupoles are used in parts of the matching sections. In ATLAS and CMS insertions, the MBWX magnets are used as D1 separation dipoles. The main parameters of these magnets are given in Table VI. In total, 45 resistive separation dipoles and $48 \mathrm{MQW}$ quadrupoles are required for the LHC insertions.

The most critical and advanced resistive magnet is the MQW twin-aperture quadrupole, featuring two $46 \mathrm{~mm}$ apertures separated by $222 \mathrm{~mm}$. It is designed and produced in collaboration with TRIUMF (Canada). After a prototype in 1998, several modifications in the design and fabrication were introduced to improve the field quality of the magnet, and a pre-series quadrupole was built by Alstom (Canada), Fig. 8. The measurements performed in CERN in March 2001 have shown that the magnet performance are compatible with the LHC requirements [8], and that the series production could start. The first series MQW are expected by the end of 2001.

The resistive dipoles MBW and MBWX are designed and built in collaboration with BINP (Russia), which is also providing resistive orbit correctors and compensator dipoles for the ALICE and LHCb experimental magnets. The first series deliveries are expected at the end of 2002.

\section{OUTLOOK}

Following comprehensive R\&D programs, the construction of the LHC insertion magnets has started in industry or laboratory production facilities. Initial experience shows that industry is in general well prepared, but that strict quality control procedures must be followed for achieving stringent requirements of the LHC magnets. Excellent and constructive relations have 


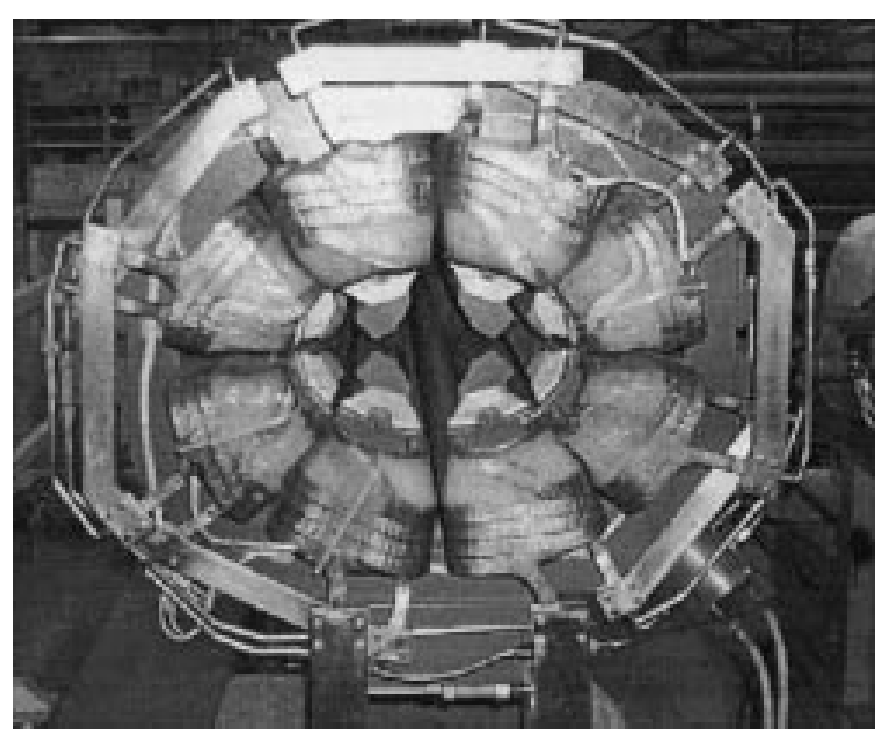

Fig. 8. Pre-series MQW twin-aperture resistive quadrupole.

been established with the accelerator laboratories, and the fabrication of equipment supplied as part of the contributions of nonmember countries is advancing well within planned delivery dates.

By their nature, the LHC insertions will very likely be upgraded following the initial years of collider operation. In this perspective, several strategies are being examined [9]. Small scale but important development effort is already pursued in the domain of accelerator grade $\mathrm{Nb}_{3} \mathrm{Sn}$ cables [10], and first designs for the second generation low- $\beta$ quadrupoles have been proposed [11]. We can therefore expect a sustained R\&D work in CERN and other laboratories in the coming years on novel magnet designs for the LHC insertions.

\section{ACKNOWLEDGMENT}

The work reported in this paper is the result of the technical expertise, ingenuity, and dedication of a number of teams and groups in CERN, in the collaborating laboratories world-wide, and in industry. The author wishes to acknowledge their excellent results, and is grateful for the contributions received.

\section{REFERENCES}

[1] The LHC design, beam performance, and machine parameters \& layouts. [Online]. Available: http://lhc.web.cern.ch/lhc.

[2] L. R. Evans, "LHC accelerator physics and technology challenges," in Proc. PAC'99, New York, March 1999, pp. 21-25.

[3] T. Shintomi et al., "Progress of the LHC low- $\beta$ quadrupole magnets at KEK," IEEE Trans. Appl. Superconductivity, vol. 11, no. 1, pp. 1562-1565, 2001.

[4] N. Andreev et al., "Status of the LHC inner triplet quadrupole program at Fermilab," IEEE Trans. Appl. Superconductivity, vol. 11, no. 1, pp. $1558-1561,2001$.

[5] E. Willen et al., "Superconducting dipole magnets for the LHC insertion regions," in Proc. EPAC'2000, Vienna, Austria, June 2000, pp. 2187-2189.

[6] J. Lucas et al., "Performance of the single and twin-aperture models of the $6 \mathrm{kA}$ superconducting quadrupole for the LHC insertions," IEEE Trans. Appl. Superconductivity, vol. 11, no. 1, pp. 1645-1648, 2001.

[7] G. Kirby et al., "Performance of the $1 \mathrm{~m}$ model of the $70 \mathrm{~mm}$ bore twin-aperture quadrupole for the LHC insertions," IEEE Trans. Appl. Superconductivity, vol. 11, no. 1, pp. 1641-1644, 2001.

[8] M. Racine et al., "Construction and measurement of the pre-series twin aperture resistive magnets for the LHC beam cleaning insertions," IEEE Trans. Appl. Superconduct., submitted for publication.

[9] K. Hubner, "Status of CERN," in Proc. HEACC-2001, Tsukuba, Japan, March 2001, CERN SL-2001-008 DI.

[10] A. den Ouden et al., "Status of the development of an $88 \mathrm{~mm}$ bore 10 T Nb3Sn model dipole magnet," IEEE Trans. Appl. Superconduct., submitted for publication.

[11] T. Sen, J. Strait, and A. V. Zlobin, "Second generation high gradient quadrupoles for the LHC interaction regions," in Proc. PAC2001, Chicago, June 2001, submitted for publication. 